\title{
Graphene Mode-Locked Femtosecond Cr:LiSAF Laser
}

\author{
F. Canbaz ${ }^{1}$, N. Kakenov ${ }^{2}$, C. Kocabas ${ }^{2}$, U. Demirbas ${ }^{3}$, and A. Sennaroglu ${ }^{1, *}$ \\ 1. Laser Research Laboratory, Departments of Physics and Electrical-Electronics Engineering, Koç University, Istanbul 34450, Turkey \\ 2. Department of Physics, Bilkent University, Ankara 06800, Turkey \\ 3. Department of Electrical and Electronics Engineering, Antalya International University, Antalya 07190, Turkey. \\ *Corresponding author: asennar@ku.edu.tr
}

\begin{abstract}
We report the first demonstration of femtosecond pulse generation from a Cr:LiSAF laser mode-locked with a monolayer graphene saturable absorber. Nearly transform-limited 72-fs pulses were generated at $850 \mathrm{~nm}$ with only two $135-\mathrm{mW}$ pump diodes.

OCIS codes: (140.4050) Mode-locked lasers; (140.5680) Rare earth and transition metal solid-state lasers; (140.7090) Ultrafast lasers; (140.3600) Lasers, tunable; (140.3480) Diode-pumped lasers; (140.3580) Solid-state lasers; (160.4236) Nanomaterials.
\end{abstract}

\section{Introduction}

The Cr:LiSAF laser, with a broad tuning range that covers the 780-1100 nm wavelength range, has been used as an efficient source of femtosecond pulses near $850 \mathrm{~nm}$ [1]. To date, pulses as short as $10 \mathrm{fs}$ and mode-locked tuning over the 800-920 $\mathrm{nm}$ wavelength range have been demonstrated [2].One drawback of the Cr:LiSAF gain medium, however, is the low nonlinear refractive index $\left(0.8 \times 10^{-16} \mathrm{~cm}^{2} / \mathrm{W}\right.$, four times lower than that for Ti:sapphire [3]), which makes it difficult to obtain a sufficient modulation depth during Kerr lens mode locking (KLM) alone. To increase the effective modulation depth for stable mode locking, numerous alternative schemes have been proposed, including gain-matched output couplers (GMOC) [4], semiconductor saturable absorber mirrors (SESAM) [5], and single-walled carbon nanotubes (SWCNT) [6]. In the case of SESAMs and SWCNTs, the operation bandwidth of the device is limited due to the resonant nature of the absorber, limiting the obtainable pulsewidths and the modelocked tuning range. Different from these schemes, mode locking can be alternatively initiated with a graphene saturable absorber (GSA) which provides saturable absorption over a wavelength range far broader than the emission band of the Cr:LiSAF gain medium [7]. However, because the optical gain of Cr:LiSAF is relatively low, a low-threshold resonator architecture with tight focusing geometry, and low-loss, dispersion optimized optics is necessary to overcome the small-signal loss of the GSA (around 5\% per round trip), especially in systems pumped by low-power single-mode diodes. In previous studies, GSA has been used to generate mode-locked pulses from bulk solid-state lasers between 800 and $2500 \mathrm{~nm}[8,9]$.

Here, we report, for the first time to our knowledge, a GSA mode-locked femtosecond Cr:LiSAF laser at $850 \mathrm{~nm}$. The Cr:LiSAF resonator with tight focusing geometry could be operated with only two 135-mW, 660-nm low-cost single-mode diode lasers. Above $135 \mathrm{~mW}$ of input pump power, continuous-wave (cw) mode locking could be initiated. At the maximum pump power of $268 \mathrm{~mW}$, the laser produced 72 -fs, nearly transform-limited pulses with an output power of $10.5 \mathrm{~mW}$ at the center wavelength of $850 \mathrm{~nm}$. The repetition rate was measured to be $132 \mathrm{MHz}$, corresponding to a pulse energy and peak power of $79 \mathrm{pJ}$ and $1.1 \mathrm{~kW}$, respectively. Once mode locking was initiated with the GSA, stable, uninterrupted femtosecond pulse generation could be sustained for hours.

\section{Experimental Setup and Results}
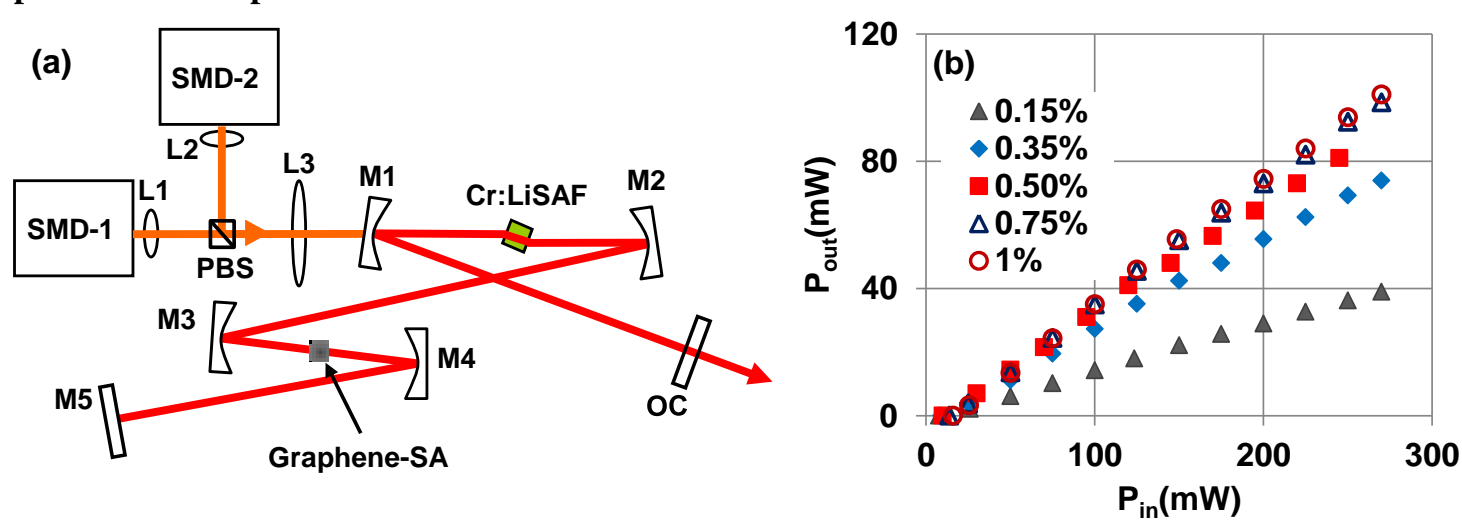

Fig. 1: (a) Schematic of the single-mode diode pumped GSA mode-locked Cr:LiSAF laser. (b) Measured variation of the laser output power as a function of the incident pump power at different output coupling levels for the free-running (without GSA) cavity. 
A schematic of the GSA mode-locked femtosecond Cr:LiSAF laser is shown in Fig. 1. The output beams of the two single-mode pump diodes (SMD-1 and SMD-2 in Fig. 1) were polarization coupled with a polarizing beam splitter (PBS) and were focused with an input lens (L3, focal length=60 mm) inside the gain crystal. The Brewster-cut, 7mm-long Cr:LiSAF gain crystal had a total absorption of $98 \%$ at $660 \mathrm{~nm}$. The astigmatically compensated x-cavity contained two curved high reflectors (M1 and M2, radius of curvature $=75 \mathrm{~mm}$ ), the Cr:LiSAF crystal between M1 and M2, a flat end high reflector (M5), and an output coupler (OC). Furthermore, the GSA, which was synthesized by chemical vapor deposition and transferred onto an infrasil substrate, was positioned at Brewster incidence in between two additional curved high reflectors (M3 and M4, radius of curvature $=75 \mathrm{~mm}$ ). The total cavity length was $112 \mathrm{~cm}$, corresponding to a pulse repetition rate of $132 \mathrm{MHz}$. Figure 1 (b) shows the variation of the cw output power as a function of the pump power (free-running resonator) for different output coupling levels. With a total pump power of $268 \mathrm{~mW}$, as high as $101 \mathrm{~mW}$ of output power was obtained by using the $1 \%$ transmitting output coupler. Since the inclusion of the GSA provides additional losses of about $2.3 \%$ per transit, the output coupler with $0.5 \%$ transmission was used in the mode locking experiments to increase the intracavity intensity.

In the experiments, the ultrafast dynamics and absorption saturation behavior of the GSA were first investigated with a commercial time-resolved pump-probe spectrometer (HELIOS, Ultrafast systems). Two-color pump-probe measurement was performed by using two tunable optical parametric oscillators (TOPAS, Newport Spectra Physics), which provided the pump $(850 \mathrm{~nm})$ and probe $(950 \mathrm{~nm})$ beams. Figure 2 (a) shows the variation of the measured fractional transmission $(\Delta T / T)$ of the probe as a function of delay. The fast and slow decay times of the GSA were determined to be $128 \mathrm{fs}$ and $1.630 \mathrm{ps}$, respectively, in good agreement with the values previously reported in the literature [10]. From the saturation data shown in Fig. 2 (b), the best-fit values of the saturation fluence and modulation depth were further determined to be $28 \mu \mathrm{J} / \mathrm{cm}^{2}$ and $0.62 \%$, respectively.
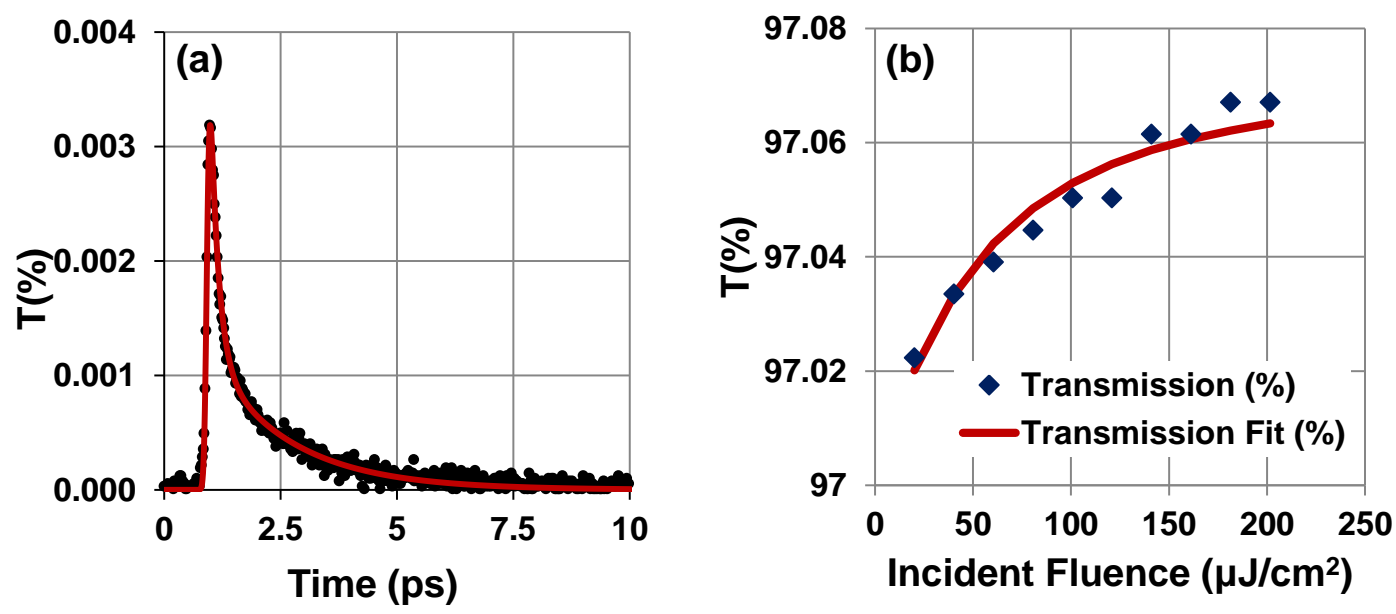

Fig. 2. (a)Measured variation of the fractional transmission ( $\Delta T / T)$ of the probe $(950 \mathrm{~nm})$ as a function of delay. (b)

Measured variation of the probe transmission as a function of the incident fluence at the wavelength of $850 \mathrm{~nm}$ for the GSA.

Once the GSA was inserted into the Cr:LiSAF resonator between the curved mirrors M3 and M4, mode locking could be readily obtained by translating the output coupler. The optical spectrum of the mode-locked output, shown in Fig. 3 (a), was recorded at the input power of $268 \mathrm{~mW}$. The output coupler transmission was $0.5 \%$. Interferometric autocorrelation measurement yielded a pulse duration of $72 \mathrm{fs}$ by assuming a sech ${ }^{2}$ intensity profile. The maximum mode-locked output power was $10.5 \mathrm{~mW}$ with a pulse repetition rate of $132 \mathrm{MHz}$. The corresponding maximum fluence on the GSA was $560 \mu \mathrm{J} / \mathrm{cm}^{2}$, suggesting that based on our saturation measurements described above, the GSA was fully saturated. Stable cw mode-locked operation could be initiated at any pump power above $120 \mathrm{~mW}$ (corresponding output power=4.2 $\mathrm{mW}$ ). As the input power was decreased, initiation of mode-locking became more difficult. Below $120 \mathrm{~mW}$ of pump power, stable pulsed operation could not be initiated. In this regime, although Q-switching tendency was observed by translating the output coupler mirror, stable Q-switching could not be observed. Based on the comparison of the threshold pump powers with and without the GSA in the Cr:LiSAF resonator, the insertion loss of the GSA was estimated to be $4.7 \%$.

To obtain solitary pulses, dispersion management was employed by using commercial and custom-made dispersion control optics (M1-M2 each with $-80 \pm 10 \mathrm{fs}^{2}$ of group delay dispersion (GDD) per bounce; M5 with$40 \pm 10 \mathrm{fs}^{2}$ of GDD per bounce). The material dispersion contributions of the gain crystal and infrasil substrate were $+24 \mathrm{fs}^{2}$ and $40 \mathrm{fs}^{2}$ per $\mathrm{mm}$, respectively. Because the net GDD of the cavity was close to zero, it was not possible to 

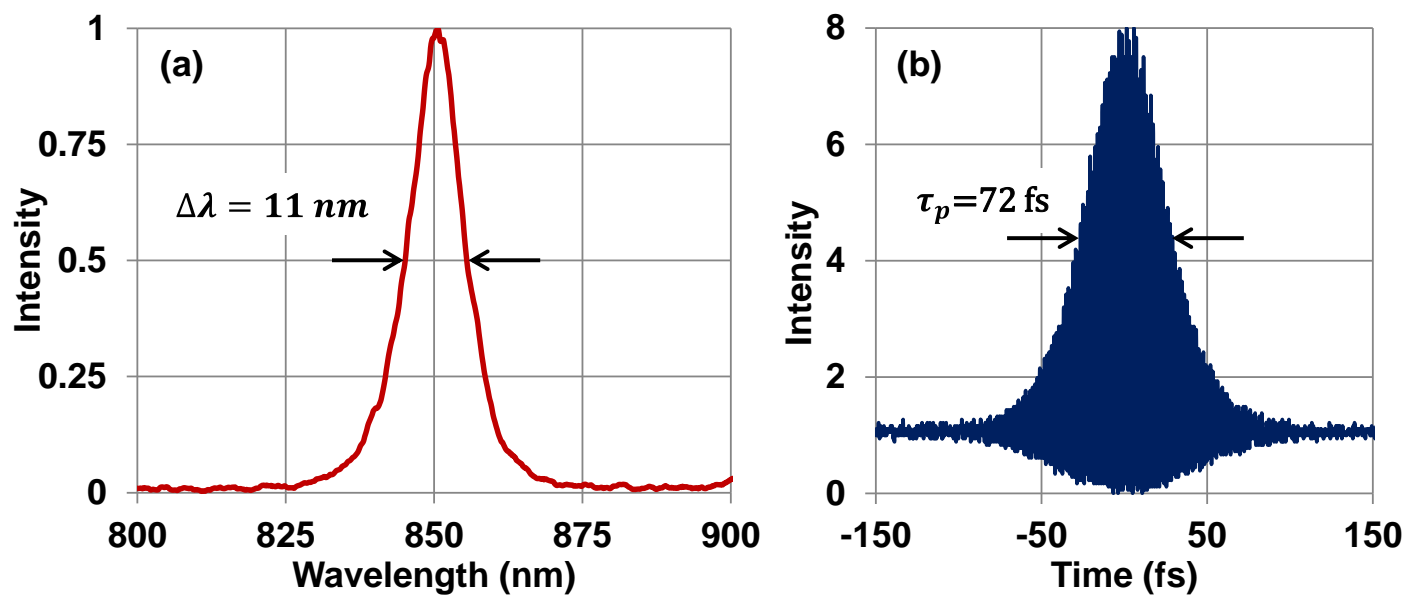

Fig. 3: (a) Measured optical spectrum of the mode-locked output at the input pump power of $268 \mathrm{~mW}$. The spectral bandwidth (FWHM) was measured to be $11 \mathrm{~nm}$. (b) Measured interferometric autocorrelation trace of the 72-fs pulses.

determine the overall dispersion from the specified GDD values given above due to the dependence of the actual GDD values on the angle of incidence. Instead, we estimated the net cavity GDD by using the soliton area theorem which relates the pulse duration to the cavity dispersion (D), intracavity pulse energy (W), and the nonlinearity coefficient $(\delta)$ through the following equations [11]:

$$
\tau=\frac{4|\mathrm{D}|}{\mathrm{W} \delta} \quad \text { (1) } \quad \delta=\frac{2 \pi \mathrm{L} n_{2}}{\lambda \mathrm{A}_{\mathrm{eff}}}
$$

Here, $n_{2}$ is the nonlinear refractive index $\left(0.8 \times 10^{-20} \mathrm{~m}^{2} / \mathrm{W}\right.$ for Cr:LiSAF $)$ [3], $\tau$ is the pulse width divided by 1.76 , $|\mathrm{D}|$ refers to the magnitude of the net round-trip GDD, L is the length of the Cr:LiSAF crystal, $\lambda$ is the output wavelength, and $A_{\text {eff }}$ is the effective beam area inside the gain medium. Based on this analysis, the net roundtrip cavity GDD was estimated to be $-75 \mathrm{fs}^{2}$ during mode-locked operation.

\section{Conclusions}

In conclusion, we reported a mode-locked femtosecond Cr:LiSAF laser, initiated with a high quality monolayer graphene saturable absorber. With only two $135-\mathrm{mW}$, 660-nm single-mode diode lasers, the low-threshold laser produced stable, nearly transform-limited, 72-fs pulses at $850 \mathrm{~nm}$ with an average power of $10.5 \mathrm{~mW}$.

This project was supported by the Scientific and Technological Research Council of Turkey (TUBITAK) under the projects $112 \mathrm{~T} 967$ and $114 \mathrm{~F} 185$.

\section{References}

[1] G. J. Valentine, J. M. Hopkins, P. LozaAlvarez, G. T. Kennedy, W. Sibbett, D. Burns, and A. Valster, "Ultralow-pump-threshold, femtosecond $\mathrm{Cr}^{3+}: \mathrm{LiSrAlF}_{6}$ laser pumped by a single narrow-stripe AlGaInP laser diode," Opt. Lett. 22, 1639-1641, (1997).

[2] C. Cihan, E. Beyatli, F. Canbaz, L. J. Chen, B. Sumpf, G. Erbert, A. Leitenstorfer, F. X. Kartner, A. Sennaroglu, and U. Demirbas, "Gain-Matched Output Couplers for Efficient Kerr-Lens Mode-Locking of Low-Cost and High-Peak Power Cr:LiSAF Lasers," IEEE J. Sel. Top. Quant. 21, (2015).

[3] E. Sorokin, "Solid-state materials for few-cycle pulse generation and amplification," in Few-cycle laser pulse generation and its applications, vol. 95, Topics in Applied Physics, F. X. Kartner, Ed. Berlin: Springer-Verlag, 2004, pp. 3-71.

[4] F. Canbaz, E. Beyatli, L. J. Chen, A. Sennaroglu, F. X. Kartner, and U. Demirbas, "Highly efficient and robust operation of Kerr-lens mode-locked Cr:LiSAF lasers using gain-matched output couplers," Opt. Lett. 39, 327-330, (2014).

[5] D. Kopf, K. J. Weingarten, G. Zhang, M. Moser, M. A. Emanuel, R. J. Beach, J. A. Skidmore, and U. Keller, "High-average-power diode-pumped femtosecond Cr:LiSAF lasers," Appl. Phys. B-Lasers O. 65, 235-243, (1997).

[6] A. Agnesi, F. Pirzio, E. Ugolotti, S. Y. Choi, D. I. Yeom, and F. Rotermund, "Femtosecond single-mode diode-pumped Cr:LiSAF laser mode-locked with single-walled carbon nanotubes," Opt. Commun. 285, 742-745, (2012).

[7] A. B. Kuzmenko, E. van Heumen, F. Carbone, and D. van der Marel, "Universal optical conductance of graphite," Phys. Rev. Lett. 100, (2008).

[8] I. H. Baek, H. W. Lee, S. Bae, B. H. Hong, Y. H. Ahn, D. I. Yeom, and F. Rotermund, "Efficient Mode-Locking of Sub-70-fs Ti:Sapphire Laser by Graphene Saturable Absorber," Appl. Phys. Express 5, (2012).

[9] M. N. Cizmeciyan, J. W. Kim, S. Bae, B. H. Hong, F. Rotermund, and A. Sennaroglu, "Graphene mode-locked femtosecond Cr:ZnSe laser at $2500 \mathrm{~nm}, "$ Opt. Lett. 38, 341-343, (2013).

[10] J. M. Dawlaty, S. Shivaraman, M. Chandrashekhar, F. Rana, and M. G. Spencer, "Measurement of ultrafast carrier dynamics in epitaxial graphene," Appl. Phys. Lett. 92, 042116, (2008).

[11] H. A. Haus, "Mode-locking of lasers," IEEE J. Sel. Top. Quant. 6, 1173-1185, (2000). 